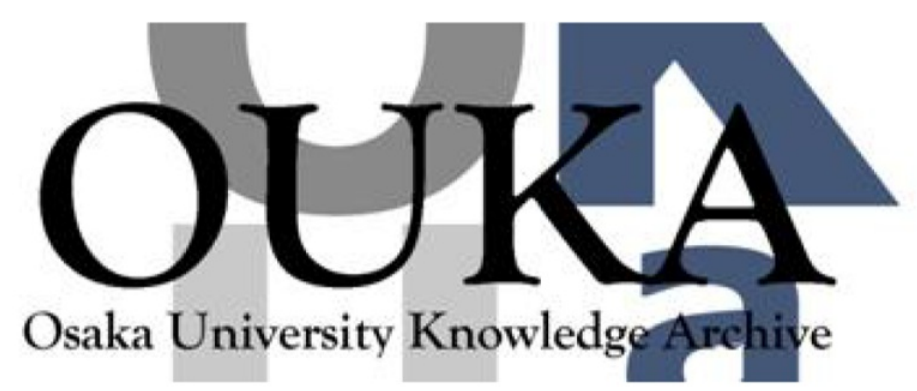

\begin{tabular}{|c|c|}
\hline Title & $\begin{array}{l}\text { Lyotropic liquid crystallinity of amy lose } \\
\text { tris(alkylcarbamates): Cholesteric and smectic } \\
\text { phase formation in different solvents }\end{array}$ \\
\hline Author (s) & $\begin{array}{l}\text { Oyamada, Keiko; Terao, Ken; Suwa, Masayori et } \\
\text { al. }\end{array}$ \\
\hline Citation & Macromolecules. 46(11) p. 4589-p. 4595 \\
\hline Issue Date & $2013-05-20$ \\
\hline oaire:version & AM \\
\hline URL & https://hdl. handle. net/11094/81836 \\
\hline rights & $\begin{array}{l}\text { This document is the Accepted Manuscript } \\
\text { version of a Published Work that appeared in } \\
\text { final form in Macromolecules, } \odot \text { American } \\
\text { Chemical Society after peer review and } \\
\text { technical editing by the publisher. To access } \\
\text { the final edited and published work see } \\
\text { https://doi.org/10.1021/ma400787c. }\end{array}$ \\
\hline Note & \\
\hline
\end{tabular}

Osaka University Knowledge Archive : OUKA

https://ir. Library. osaka-u. ac. jp/

Osaka University 


\title{
Lyotropic Liquid Crystallinity of Amylose Tris(alkylcarbamates): Cholesteric and Smectic Phase Formation in Different Solvents
}

\author{
Keiko Oyamada, ${ }^{\dagger}$ Ken Terao, ${ }^{* \dagger}$ Masayori Suwa, ${ }^{\ddagger}$ Shinichi Kitamura ${ }^{\S}$ Takahiro Sato ${ }^{\dagger}$ \\ †Department of Macromolecular Science, Graduate School of Science, Osaka University, 1-1 \\ Machikaneyama-cho, Toyonaka, Osaka 560-0043, Japan. \\ Department of Chemistry, Graduate School of Science, Osaka University, 1-1 Machikaneyama- \\ cho, Toyonaka, Osaka 560-0043, Japan. \\ ${ }^{\S}$ Graduate School of Life and Environmental Sciences, Osaka Prefecture University, Gakuen-cho, \\ Nakaku, Sakai, 599-8531, Japan. \\ * Corresponding Author. E-mail: kterao@chem.sci.osaka-u.ac.jp \\ Dedicated to the memory of the late Professor Akio Teramoto.
}

ABSTRACT: Lyotropic liquid crystallinity was investigated for amylose tris( $n$-butylcarbamate) (ATBC), amylose tris(ethylcarabamate) (ATEC), and amylose tris ( $n$-hexylcarbamate) (ATHC) in tetrahydrofuran (THF) and ethyl lactates (ELs) by using phase separation experiments, polarized microscopic observation, small-angle X-ray diffraction, and circular dichroism measurements. Concentrated THF solution of ATBC has selective reflection at visible light wavelength, indicating the formation of cholesteric phase. A current theory well explains the phase diagram of ATBC, ATEC, and ATHC in THF. On the other hand, ELs solution forms smectic phase and it has significantly different phase diagram, that is, the biphasic range is much wider than that in THF. In other words, highly concentrated smectic phase can be obtained from semidilute and rather low viscosity ELs solution. This is likely because amylose alkylcarbamate chains may have significant anisotropic intermolecular attractive interaction in between polymer chains in ELs or intermolecular interactions between the chain ends are much more different from those in THF.

\section{- INTRODUCTION}

As many biopolymers have rather stiff main chain in solution, their high concentrated solutions may form lyotropic mesophase. ${ }^{1}$ Such lyotropic liquid crystalline phase was also found for single stranded polysaccharides and their derivatives, that is, cellulose, ${ }^{2-12}$ chitosan, ${ }^{13-14}$ and curdlan. ${ }^{15}$ Among them, polysaccharide phenylcarbamates have good solubility in many organic solvents including some theta solvents ${ }^{16}$ whereas they behave as rather stiff chains. ${ }^{16-21}$ Thus, they are suitable to investigate lyotropic liquid crystallinity, and indeed, quantitative discussions have been reported for cellulose tris(phenylcarbamate) (CTPC) ${ }^{20}$ and chitosan phenylcarbamate. ${ }^{14}$

Amylose is a component of starch which is the second abundant biomass but few reports are found for its lyotropic liquid crystallinity. ${ }^{22}$ Indeed, liquid crystallinity of amylose tris(phenylcarbamate) (ATPC) is difficult to investigate owing to the extremely high viscosity of the concentrated solution even though ATPC has relatively high chain stiffness in dilute solution: ${ }^{17}$ The chain stiffness parameter $\lambda^{-1}$ (the Kuhn segment length, twice of the persistence length) is $22 \mathrm{~nm}$ in 1,4-dixane. ${ }^{21}$ On the other hand, we recently reported that three amylose 
alkyl carbamates, that is, amylose tris( $n$-butylcarbamate) (ATBC), amylose tris(ethylcarbamate) (ATEC), and amylose tris( $n$-hexylcarbamate) (ATHC) of which chemical structures are illustrated in Chart 1 have larger $\lambda^{-1}$ values in rather less polar solvents, and hence they form tightly wound rigid helices stabilized by intramolecular hydrogen bonds. ${ }^{23-26}$ In actuality, concentrated solutions of ATBC showed liquid crystallinity in tetrahydrofuran (THF), $\mathrm{D}^{-}$and $\mathrm{L}^{-}$ ethyl lactates ( $\mathrm{D}^{-}$and $\mathrm{L}^{-E L}$ ), 2-butanol, 2-ethoxyethanol, 1-propanol, and 2-propanol. In the present study we chose THF and $\mathrm{D}^{-}$and $\mathrm{L}_{\mathrm{L}}$-EL since ATBC in THF has the highest $\lambda^{-1}$ in these solvents $^{23,25-26}$ (Table 1), and biphasic phase for ATBC in $\mathrm{D}^{-}$and $\mathrm{L}_{\mathrm{L}}$-EL were found even at lower concentrations $\left(<0.2 \mathrm{~g} \mathrm{~cm}^{-3}\right)$ though $\lambda^{-1}$ in ELs is appreciably lower than that in THF. ${ }^{26}$ This suggests that ATBC in THF forms different liquid crystal phases from that in ELs. This may be possible because both nematic and smectic phases were found for narrow dispersed polymer in concentrated solution, ${ }^{27-28}$ but solvent dependent liquid crystal phase is rarely found probably because most of stiff polymer chains soluble in limited solvents. We thus determined phase diagram as well as liquid crystal structure, that is, selective reflection and X-ray diffraction in THF and $\mathrm{D}^{-}$and $\mathrm{L}_{\mathrm{E}} \mathrm{EL}$.

Chart 1. Chemical Structures of Amylose Tris(ethylcarbamate) (1. ATEC), Amylose Tris(n-butylcarbamate) (2. ATBC), and Amylose Tris(n-hexylcarbamate) (3. ATHC).

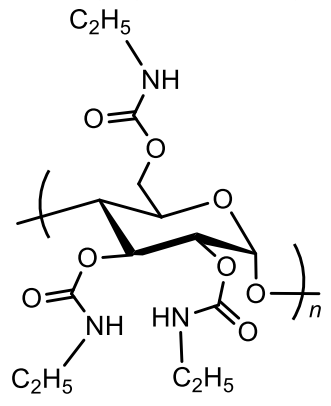

1. ATEC

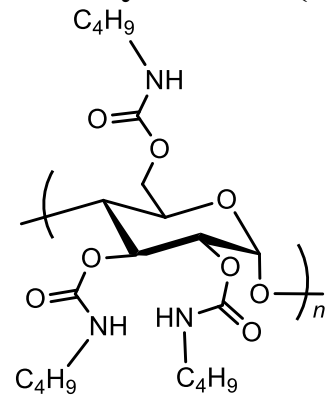

2. ATBC

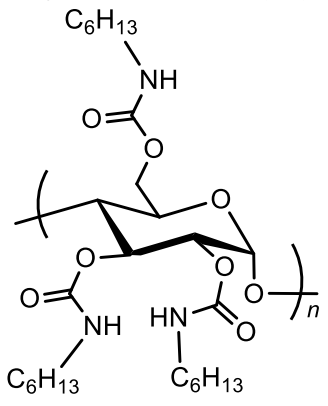

3. ATHC

Table 1. Wormlike Chain Parameters (Helix Pitch per Residue $h$ and the Kuhn Segment Length $\lambda^{-1}$ ) of ATBC, ATEC, and ATHC in THF and $\mathrm{D}^{-}$and $\mathrm{L}_{\text {-Ethyl Lactates (D-EL and } \mathrm{L}^{-}}$ EL).

\begin{tabular}{cccc}
\hline Polymer & Solvent & $h(\mathrm{~nm})$ & $\lambda^{-1}(\mathrm{~nm})$ \\
\hline ATBC & THF & $0.26^{\mathrm{a}}$ & $75^{\mathrm{a}}$ \\
ATBC & D-EL & $0.26^{\mathrm{b}}$ & $49^{\mathrm{b}}$ \\
ATBC & L-EL & $0.26^{\mathrm{b}}$ & $32^{\mathrm{b}}$ \\
ATEC & THF & $0.36^{\mathrm{c}}$ & $33^{\mathrm{c}}$ \\
ATEC & D-EL & $0.35^{\mathrm{c}}$ & $27^{\mathrm{c}}$ \\
ATHC & THF & $0.29^{\mathrm{c}}$ & $75^{\mathrm{c}}$ \\
\hline
\end{tabular}

${ }^{a}$ Reference ${ }^{23} \cdot{ }^{b}$ Reference ${ }^{26} \cdot{ }^{c}$ Reference ${ }^{24}$. 


\section{- EXPERIMENTAL SECTION}

Samples and Solvents. Previously investigated 3 ATEC, 6 ATBC, and 3 ATHC samples ${ }^{23-24}$ obtained from enzymatically synthesized amylose of which main chain length has relatively narrow dispersity and no branch were used for this study. Three samples (ATBC60K, ATBC140K, and ATHC130K) were further prepared in this study with the same method reported previously. The weight-average and number-average molar masses $\left(M_{\mathrm{w}}\right.$ and $\left.M_{\mathrm{n}}\right)$ were determined from conventional static light scattering measurements and size-exclusion chromatography equipped with multi-angle laser-light scattering. The values of $M_{\mathrm{w}}$ are summarized in Table 2 along with those for dispersity indices (DI) defined as the ratio of $M_{\mathrm{w}}$ to $M_{\mathrm{n}}$ or the ratio of the $z$-average molar mass $M_{\mathrm{z}}$ to $M_{\mathrm{w}}$. Commercially available THF and L-ethyl lactate (L-EL) were purified by fractional distillation and ${ }_{D}$-ethyl lactate (D-EL) was prepared from $\mathrm{D}$-methyl lactate in the manner reported in ref. ${ }^{26}$.

Table 2. Weight-Average Molar Mass $M_{\mathrm{w}}$ and Dispersity Index (DI) of the Samples

\begin{tabular}{|c|c|c|c|c|c|}
\hline Sample & $\begin{array}{c}10^{-3} M_{\mathrm{w}} \\
\left(\mathrm{g} \mathrm{mol}^{-1}\right)\end{array}$ & DI & Sample & $\begin{array}{c}10^{-3} M_{\mathrm{w}} \\
\left(\mathrm{g} \mathrm{mol}^{-1}\right)\end{array}$ & DI \\
\hline ATBC $17 \mathrm{~K}^{\mathrm{a}}$ & 16.6 & $1.03^{\mathrm{d}}$ & ATEC $32 \mathrm{~K}^{\mathrm{b}}$ & 31.9 & $1.05^{\mathrm{e}}$ \\
\hline ATBC60K ${ }^{c}$ & 60.3 & $1.05^{\mathrm{d}}$ & ATEC $150 K^{b}$ & 154 & $1.11^{\mathrm{e}}$ \\
\hline ATBC $110 K^{a}$ & 105 & $1.05^{\mathrm{e}}$ & ATEC $400 K^{b}$ & 390 & $1.06^{\mathrm{d}}$ \\
\hline $\mathrm{ATBC} 130 \mathrm{~K}^{\mathrm{a}}$ & 133 & $1.02^{\mathrm{e}}$ & ATHC49K & 48.8 & $1.18^{\mathrm{e}}$ \\
\hline $\mathrm{ATBC} 140 \mathrm{~K}^{\mathrm{c}}$ & 141 & $1.06^{\mathrm{d}}$ & $\mathrm{ATHC} 130 \mathrm{~K}^{\mathrm{c}}$ & 129 & $1.02^{\mathrm{d}}$ \\
\hline $\mathrm{ATBC} 250 \mathrm{~K}^{\mathrm{a}}$ & 255 & $1.07^{\mathrm{d}}$ & ATHC $300 \mathrm{~K}^{\mathrm{b}}$ & 286 & $1.03^{\mathrm{d}}$ \\
\hline ATBC $260 K^{a}$ & 263 & $1.06^{\mathrm{d}}$ & ATHC $460 \mathrm{~K}^{\mathrm{b}}$ & 457 & $1.18^{\mathrm{d}}$ \\
\hline ATBC $490 K^{a}$ & 490 & $1.02^{\mathrm{d}}$ & & & \\
\hline
\end{tabular}

Phase Separation Measurements. Concentrated solutions were prepared in a calibrated glass tube at $40{ }^{\circ} \mathrm{C}$ and the biphasic solution was centrifuged at $5,000 \mathrm{rpm}$ at $25^{\circ} \mathrm{C}$ for one to three days to achieve a complete phase separation. The tube was placed to the water bath thermo-stated at $25^{\circ} \mathrm{C}$ to determine the volume fraction of liquid crystal phase $\Phi_{\mathrm{LC}}$ by using a travelling microscope. The measurements were performed for different polymer mass concentration $c$ to determine $\Phi_{\mathrm{LC}}$ as a function of the initial concentration $c_{0}$ for ATBC60K, ATBC110K, ATBC260K, ATEC32K, ATEC49K, ATHC130K, ATHC300K, and ATHC460K in THF. These measurements were also carried out for ATBC60K and ATBC110K in $\mathrm{D}^{-}$and $\mathrm{L}_{\mathrm{L}} \mathrm{EL}$ and ATBC260K in D-EL. The samples were dissolved at $40-60{ }^{\circ} \mathrm{C}$. From the obtained relationship between $c_{0}$ and $\Phi_{\mathrm{LC}}$, the phase boundary concentration $c_{\mathrm{I}}$ between the isotropic phase and biphasic region and that $c_{\mathrm{A}}$ between the biphasic region and the anisotropic phase (Figure 1a). Since complete phase separation was not achieved for ATBC17K, ATBC490K, ATEC150K, and ATEC400K in THF owing to high viscosity, the biphasic solution was warmed to obtain isotropic phase and cooled gradually in the thermo-stated water bath to find the 
temperature where the optical birefringence of the solution appears using two polarizing plates arranged in a crossed-Nicol configuration. The obtained $c_{\mathrm{I}}$-temperature relationships are shown in Figure $1 \mathrm{~b}$, to estimate $c_{\mathrm{I}}$ at $25^{\circ} \mathrm{C}$.
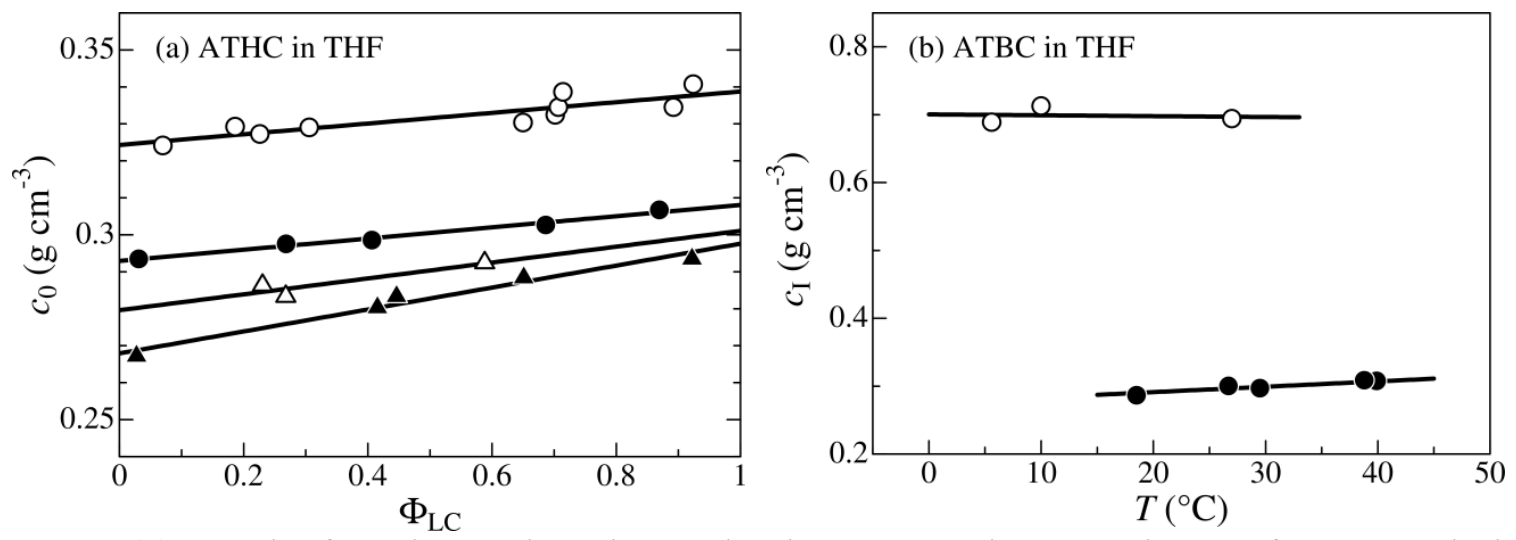

Figure 1. (a) Results from isotropic-anisotropic phase separation experiments for THF solutions of ATHC49K (unfilled circles), ATHC130K (filled circles), ATHC300K (unfilled triangles), and ATHC460K (filled triangles) at $25^{\circ} \mathrm{C}$. (b) Temperature dependence of $c_{\text {I }}$ for ATBC17K (unfilled circles) and ATBC490K (filled circles) in THF.

Selective Reflection of Circularly Polarized Light. Selective reflection measurements of circularly polarized light were made for ATBC130K and ATBC260K in THF using a JASCO J720WO spectropolarimeter equipped with a Peltier thermostated cell holder and a rectangular cell having $10 \mathrm{~mm}$ path length to determine ellipticity $\theta$ as functions of the wavelength $\lambda_{0}$ in vacuum, $T$, and $c$. The concentrated solutions were filled in the cell with a thick needle and each measurement was started at least 10 min after the temperature setting. It should be noted that substantially the same spectra was obtained after $30 \mathrm{~min}$, indicating the equilibrium state. Essentially the same circular dichroism spectra were obtained at the three different points in the cell, and thus the filled solution had enough homogeneity, indicating that cholesteric microdomains should be much smaller than the optical path.

Small Angle X-ray Scattering (SAXS). Synchrotron-radiation SAXS measurements were made for ATEC32K in THF and ATBC60K, ATBC110K, ATHC49K, and ATEC32K in ELs all at $25{ }^{\circ} \mathrm{C}$ using a Rigaku R-AXIS VII imaging plate detector at the BL40B2 beamline in SPring-8 (Hyogo, Japan) or at BL-10C beamline in KEK-PF (Ibaraki, Japan). The wavelength of the incident light was $0.1 \mathrm{~nm}$ or $0.15 \mathrm{~nm}$ and the camera length was set to be $4000 \mathrm{~mm}, 2000 \mathrm{~mm}$, or $1500 \mathrm{~mm}$. Liquid crystal samples were sandwiched between two thin Kapton films with the path length of $\sim 0.5 \mathrm{~mm}$ or filled in $1.5 \mathrm{~mm}$ quartz capillary tube. The measurements were also carried out for magnetic oriented samples of ATBC140K and ATBC250K in ELs, which were prepared in a $1.5 \mathrm{~mm}$ quartz capillary in a $10 \mathrm{~T}$ magnetic field for 50 hours since it is known that some liquid crystalline polymers align under magnetic field. ${ }^{29}$ The samples were stored at low temperature $\left(\sim 0^{\circ} \mathrm{C}\right)$ and made SAXS measurements after 6 to 24 hours.

\section{- RESULTS AND DISCUSSION}

Phase Diagram in THF. Figure 2 shows the phase boundary concentrations $c_{\mathrm{I}}$ and $c_{\mathrm{A}}$ for ATBC, ATEC, and ATHC in THF at $25{ }^{\circ} \mathrm{C}$. Except for ATEC, biphasic concentration range is quite narrow and both $c_{\mathrm{I}}$ and $c_{\mathrm{A}}$ decrease with increasing $M_{\mathrm{w}}$. This is typical for lyotropic liquid 
crystals for polymers having stiff main chain. Thus, to compare the phase diagrams with CTPC in THF, ${ }^{10}$ the phase boundary concentrations are plotted in Figure 3 against the Kuhn segment number $\lambda L$ in which $L$ denotes the contour length. This parameter is related to the molar mass $M$ as $L=M h / M_{0}$, where $M_{0}$ is the molar mass of the repeat unit (459.5 $\mathrm{g} \mathrm{mol}^{-1}$ for ATBC, 375.4 $\mathrm{g} \mathrm{mol}^{-1}$ for ATEC, and $543.7 \mathrm{~g} \mathrm{~mol}^{-1}$ for ATHC). Both $c_{\mathrm{A}}$ and $c_{\mathrm{I}}$ for the amylose derivatives are lower than those for CTPC. This is reasonable because the chain stiffness of the amylose derivatives are higher than that for CTPC in THF $\left(\lambda^{-1}=21 \mathrm{~nm}\right) .{ }^{20}$ We now compare these data with the scaled particle theory (SPT) based on the wormlike spherocylinder model, which is one of the most successful theories, ${ }^{30}$ and indeed it also explains phase behavior for some other polymer-solvent systems. ${ }^{10,31-33}$ The theory allows us to calculate the phase boundary concentrations $c_{\mathrm{I}}$ and $c_{\mathrm{A}}$ as a function of $\lambda L$ when $h, \lambda^{-1}$, and the hard-core diameter $d_{0}$ are given. The obtained theoretical curves fairly reproduce the experimental data except for the lowest $M_{\mathrm{w}}$ sample of ATEC, suggesting different phase appears for this sample (see the last part of "Chain Alignment"). The last parameter $\left(d_{0}\right)$ was estimated from the curve fitting procedure to be 1.47 $\mathrm{nm}, 1.30 \mathrm{~nm}$, and $1.72 \mathrm{~nm}$ for ATBC, ATEC, and ATHC, respectively. The chain diameter $d_{\mathrm{v}}$ may also be estimated from the partial specific volume $\bar{v}$ as

$$
d_{\mathrm{v}}=\sqrt{\frac{4 M_{0} \bar{v}}{\pi N_{\mathrm{A}} h}}
$$

where $N_{\mathrm{A}}$ is the Avogadro number. The values of $d_{\mathrm{v}}$ are obtained to be $1.74 \mathrm{~nm}, 1.28 \mathrm{~nm}$, and $1.87 \mathrm{~nm}$ for ATBC, ATEC, and ATHC, respectively, which are substantially close to the corresponding $d_{0}$. Thus, we may conclude that the phase diagram of ATEC, ATBC, and ATHC in THF is explained by current theories with the wormlike chain parameters obtained from the dilute solution properties reported recently. ${ }^{23-24}$

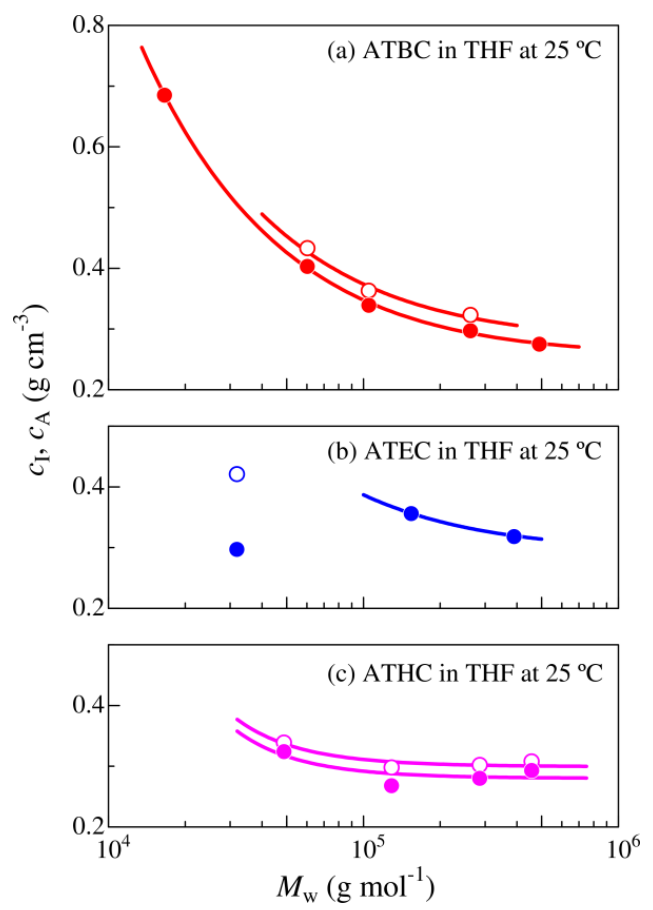

Figure 2. Molar mass dependence of $c_{\mathrm{I}}$ (filled circles) and $c_{\mathrm{A}}$ (unfilled circles) for ATBC (a), ATEC (b), and ATHC (c) in THF at $25^{\circ} \mathrm{C}$. 


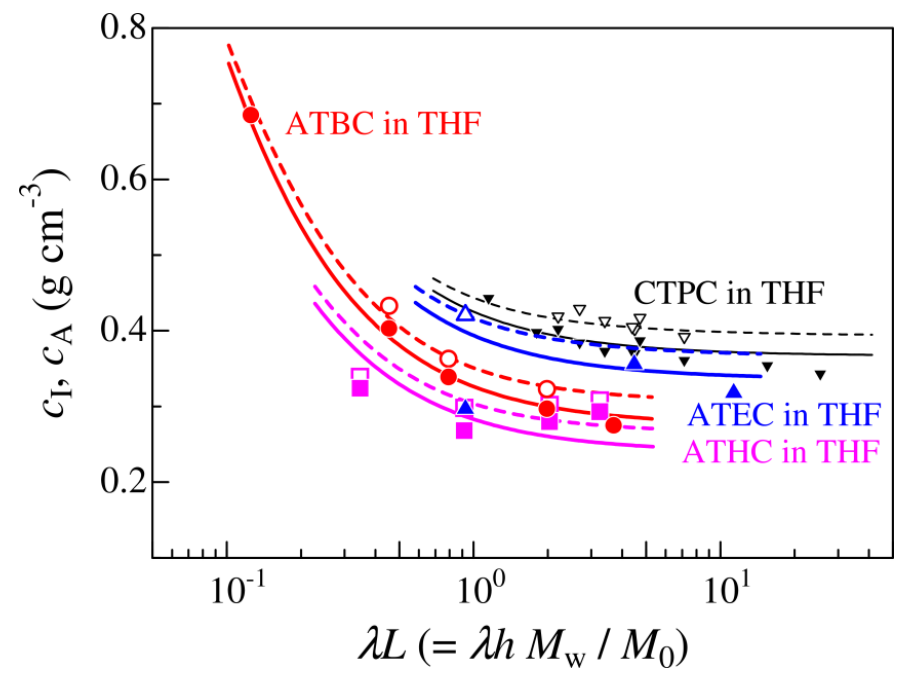

Figure 3. Comparison of experimental phase boundary concentrations for ATBC (circles), ATEC (triangles), and ATHC (squares) along with those for $\mathrm{CTPC}^{10}$ all in THF at $25^{\circ} \mathrm{C}$. Filled and unfilled symbols denote experimental $c_{\mathrm{I}}$ and $c_{\mathrm{A}}$, and solid and dashed curves are theoretical $c_{\mathrm{I}}$ and $c_{\mathrm{A}}$, respectively.

Cholesteric Pitch in THF. As shown in the TOC and our previous paper, ${ }^{23}$ liquid crystal phase of ATBC in THF shows selective reflection, indicating that the cholesteric pitch should be close to the wavelength of visible light. The cholestetic structure reflects circularly polarized light having corresponding wavelength and helix sense. Figure $4 \mathrm{a}$ shows the circular dichroism or selective reflection spectra for ATBC260K in THF at $c=0.400 \mathrm{~g} \mathrm{~cm}^{-3}$. Each spectrum has a positive peak, showing left-handed helical cholesteric structure; this helix sense is the same as that for local helical structure of ATBC. It should be noted that appreciable selective reflection was not observed for ATEC and ATHC in THF at $c \sim 0.4 \mathrm{~g} \mathrm{~cm}^{-3}$. The cholesteric pitch $P$ can be obtained from the peak wavelength $\lambda_{0, \max }$ of each spectrum to be

$$
P=-\frac{\lambda_{0, \text { max }}}{n}
$$

where $n$ denotes refractive index of the solution, and the minus sign in the right-hand side indicates that the cholesteric helix is left handed. The $P$ values are plotted against $T$ in Figures $4 \mathrm{~b}$ and $4 \mathrm{c}$ for different $c$. Their absolute values decrease with lowering temperature. Similar temperature dependence was also seen for lyotropic liquid crystals of chitosan phenylcarbamate. ${ }^{14}$ The $-P$ value decreases rapidly with increasing $c$ at the all investigated temperatures as shown in Figure 4d but the values for ATBC130K are appreciably larger than those for ATBC260K at the same $T$ and $c$. This is possibly because end effects of the polymer chain are not negligible for ATBC130K and even possibly for ATBC260K. The concentration dependence of $P$ was formulated by Sato et al. ${ }^{34}$ using the equivalent freely jointed chain model. $^{35}$ As shown in Figure 4d, the theory may explain the current concentration dependence of $P$ of each sample with appropriate chiral interaction parameters (see Supporting Information for the theoretical details and the parameters). We do not discuss the further theoretical analysis since the present study investigated only two ATBC samples. 

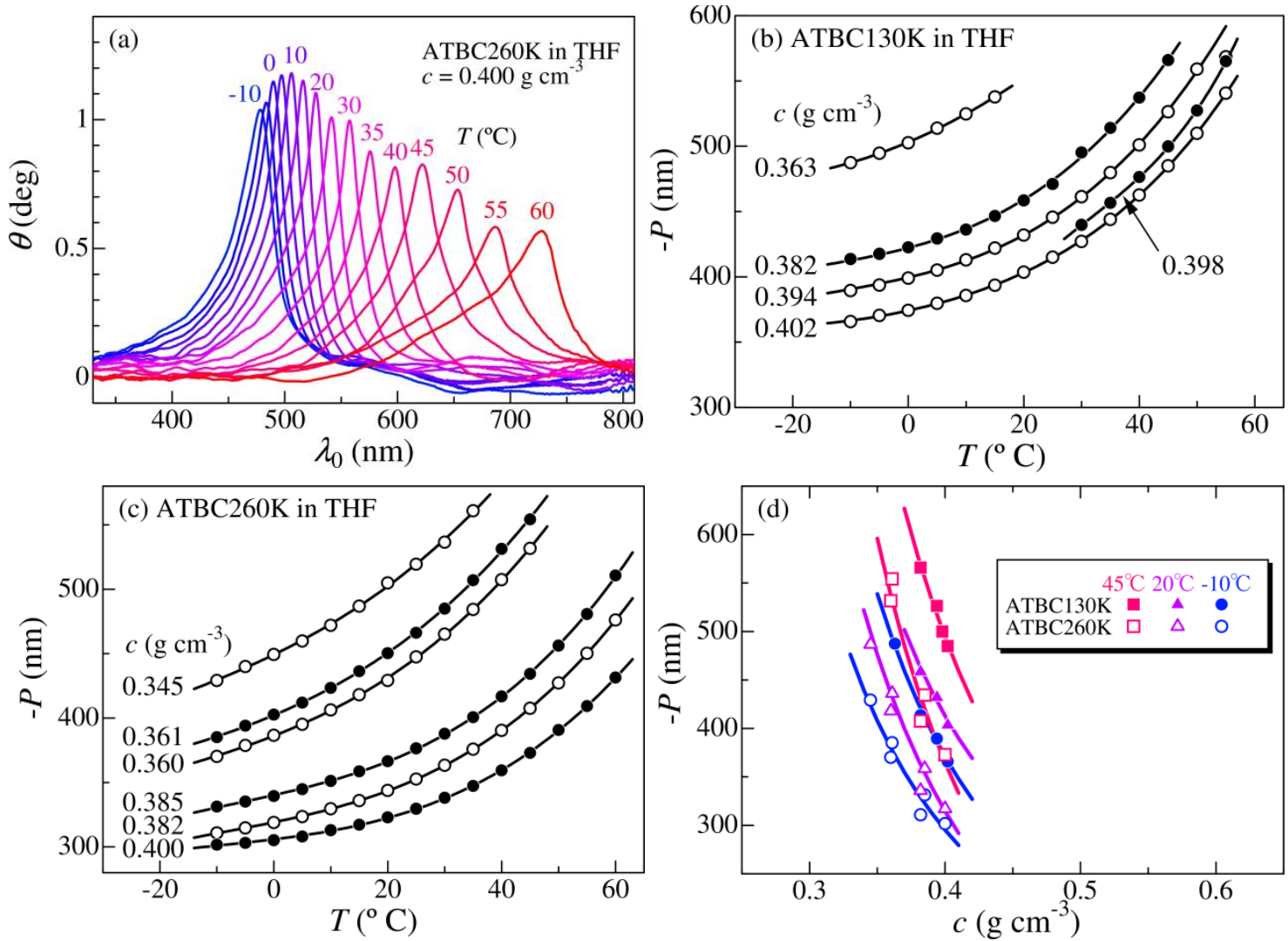

Figure 4. Cholesteric pitches $P$ for ATBC in THF. (a) Circular dichroism spectra for ATBC260K ( $\left.c=0.400 \mathrm{~g} \mathrm{~cm}^{-3}\right)$. (b) Temperature dependence of $P$ for ATBC130K at indicated $c$. (c) Temperature dependence of $P$ for ATBC260K at indicated $c$. (d) Concentration dependence of $P$ at $-10{ }^{\circ} \mathrm{C}$ (circles), $20{ }^{\circ} \mathrm{C}$ (triangles), and $45{ }^{\circ} \mathrm{C}$ (squares) for ATBC130K (filled symbols) and $\mathrm{ATBC} 260 \mathrm{~K}$ (unfilled symbols). Solid curves are the theoretical values (see Supporting Information).

Phase Diagram in Ethyl Lactates. Biphasic solutions of ATBC in ELs can be separated by centrifugation, but the concentrated phase was still turbid even after 12 hours centrifugation and the volume fraction of the turbid phase $\Phi_{\text {turbid }}$ is essentially irrespective of the duration. Plots of $c_{0}$ vs $\Phi_{\text {turbid }}$ are illustrated in Figure 5. The values of $c_{\mathrm{I}}$ and the apparent $c_{\mathrm{A}}\left(c_{\mathrm{A} \text {,app }}\right)$ can be obtained from the intercept to $\Phi_{\text {turbid }}=0$ and 1, respectively. However, from the polarized microscopic observation in Figure 6, it is found that the turbid phase consists of liquid crystal and isotropic phases, thus the actual $c_{\mathrm{A}}$ should be much higher than $c_{\mathrm{A} \text {,app }}$ obtained from the intercept to $\Phi_{\text {turbid }}=1$. Indeed, higher concentrated liquid crystalline solution $\left(c=0.55 \mathrm{~g} \mathrm{~cm}^{-3}\right.$, see Figure 6b) becomes more transparent than that at $c=0.32 \mathrm{~g} \mathrm{~cm}^{-3}$, which is still higher than $c_{\mathrm{A} \text {,app. }}$ It should be noted that typical micro-size structures for smectic phase were not found for the concentrated solution. Therefore, this stable turbidity is probably because of long time scale to merge two liquid crystalline droplets. This is called as the viscoelastic effect ${ }^{36}$ and similar behaviors are found for some other polymer-solvent systems. ${ }^{37-39}$ While this complex phenomena, we may still conclude that the obtained $c_{\mathrm{I}}$ values are much smaller and the ratios of $c_{\mathrm{A}}$ to $c_{\mathrm{I}}$ are much larger than those for ATBC-THF systems, indicating that SPT for isotropic and nematic phase separation is not applicable to the system. This is likely due to significant 
anisotropic attractive intermolecular interaction between two ATBC chains in ELs or intermolecular interactions between polymer and solvent molecules. The obtained $c_{\mathrm{I}}$ in ${ }_{\mathrm{D}}-\mathrm{EL}$ is appreciably smaller than that in ${ }_{\mathrm{L}}-\mathrm{EL}$. This might be related to the higher chain stiffness in ${ }_{\mathrm{D}}$-EL than that in ${ }_{\mathrm{L}}$-EL considering attractive interactions between two rigid rods strongly depend on the angle of the two rods. Turbid phase was also found for ATEC and ATHC in ELs and their $c_{I}$ are smaller than that in THF but it was hardly to decide the phase boundary concentrations by the centrifugation method because of less reproducibility.

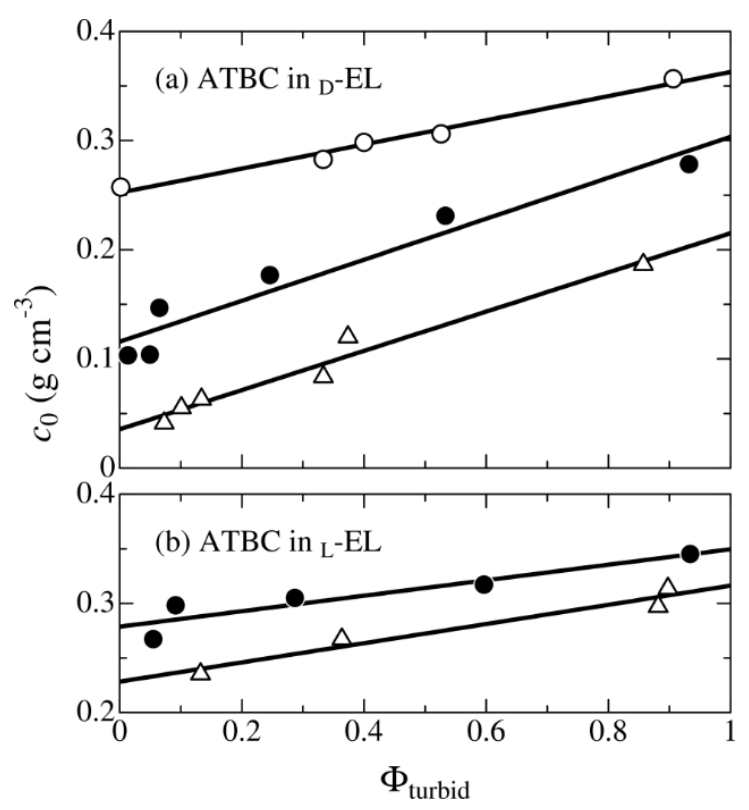

Figure 5. Results from isotropic-anisotropic (turbid) phase separation experiments for $\mathrm{D}-\mathrm{EL}$ solutions (a) for ATBC60K (unfilled circles), ATBC110K (filled circles), and ATBC260K (triangles), and for ${ }_{\mathrm{L}}$-EL solutions for $\mathrm{ATBC} 110 \mathrm{~K}$ (filled circles) and $\mathrm{ATBC} 260 \mathrm{~K}$ (triangles) all at $25^{\circ} \mathrm{C}$.

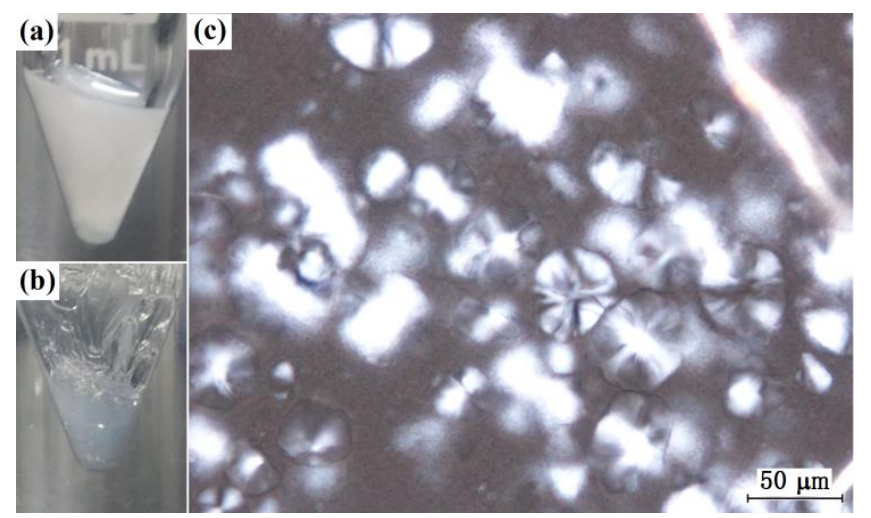

Figure 6. Photographs for ATBC130K in $\mathrm{D}-\mathrm{EL}$ at $c=0.32 \mathrm{~g} \mathrm{~cm}^{-3}$ (a) and at $c=0.55 \mathrm{~g} \mathrm{~cm}^{-3}$ (b). (c) A polarizing microscope image for ATBC250K in ${ }_{D}-E L\left(c=0.25 \mathrm{~g} \mathrm{~cm}^{-3}\right)$.

Chain Alignment. From the significantly different phase diagram in ELs, it is expected that the alignment of ATBC chains in ELs should be different from those in THF. Figure 7a displays SAXS profiles for ATBC samples in ELs. While appreciable peaks are found in each 
profile and the peak position for the same sample are equivalent in the two solvents, the diffraction peak of larger $M_{\mathrm{w}}$ sample locates at smaller $q$. Since the higher order peaks are mostly twice and three times of those for the first peak, the d-spacing is obtained to be $42 \mathrm{~nm}$ and $58 \mathrm{~nm}$ for ATBC60K and ATBC110K, respectively. These values are much smaller than those for the cholesteric pitch as shown in Figure 4 and they are fairly close to that for the weightaverage contour length $L_{\mathrm{w}}\left(=M h / M_{0}\right)$, that is, $34 \mathrm{~nm}$ and $59 \mathrm{~nm}$ for ATBC60K and ATBC110K, respectively, indicating that these samples have smectic order in ELs. Figure 7b and 7c shows SAXS profiles for ATEC32K and ATHC49K in ELs. While each peak seems to be broader than those for ATBC, the d-spacing was roughly estimated to be $\sim 20 \mathrm{~nm}$ and $\sim 40 \mathrm{~nm}$. They are also close to those for $L_{\mathrm{w}}$, that is, $28 \mathrm{~nm}$ and $31 \mathrm{~nm}$ for ATEC32K and ATHC49K when we assume $h$ for ATHC49K in ELs is the same as that in THF, suggesting they have similar liquid crystal structure as that for ATBC in ELs.
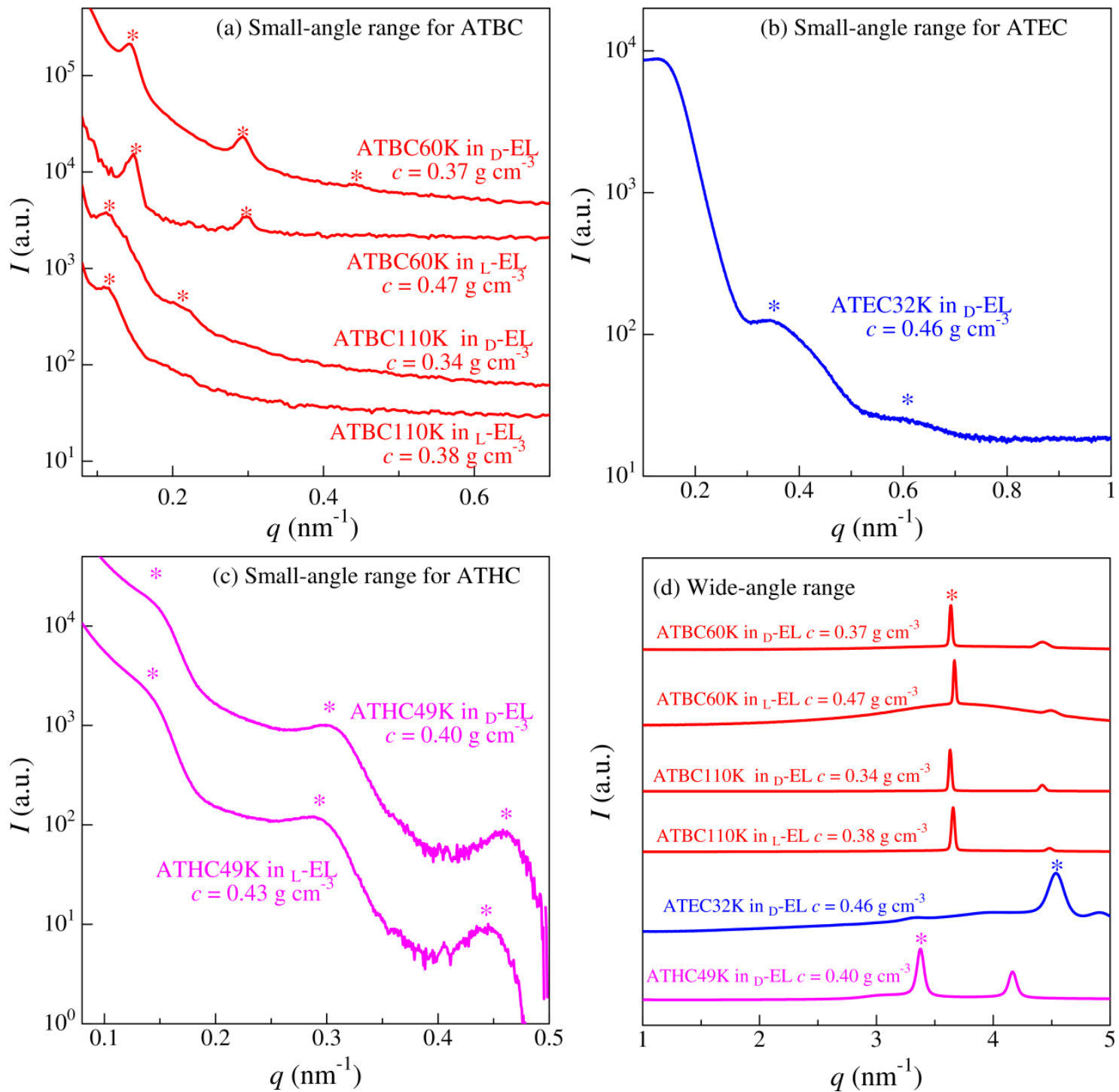

Figure 7. SAXS intensity profiles for ATBC (a), ATEC (b), ATHC (c) in ELs at $25{ }^{\circ} \mathrm{C}$. (d) Wide angle range for ATBC, ATEC, and ATHC samples in ELs at $25^{\circ} \mathrm{C}$. 
Diffraction peaks in ELs are also found at higher $q$ range. The $q$ value at the peak is substantially independent of the concentration $\left(0.42<c<0.59 \mathrm{~g} \mathrm{~cm}^{-3}\right.$, not shown here) and chirality of the solvents. This result indicates that the diffraction patterns in ELs reflects liquid crystal structure of the concentrated phase with $c=c_{\mathrm{A}}$. The peak position was obtained to be 3.65, 4.5, and $3.4 \mathrm{~nm}^{-1}$ for ATBC, ATEC, and ATHC, respectively. To assign these peaks, azimuth angle dependence of scattering intensities for magnetically aligned samples are illustrated in Figure 8. While the small angle peak at $0.077 \mathrm{~nm}^{-1}$ orients in parallel to the magnetic field, those at $3.58 \mathrm{~nm}^{-1}$ is in perpendicular, indicating that this diffraction peak reflects the packing distance between polymer chains. It should be noted that another diffraction peak was found at slightly higher $q$ at $4.34 \mathrm{~nm}^{-1}$ for some samples, but as shown in Figure $7 \mathrm{~d}$, this peak for some samples is much smaller than that at $3.6 \mathrm{~nm}^{-1}$ and furthermore, no orientation was observed in magnetically aligned sample in Figure 8. Similar behavior was also found for ATBC250K (not shown here). Thus, the peak may reflect different structure nearby the interface of the capillary cell. Diffraction peaks are also found for ATBC60K in ${ }_{\mathrm{L}}$-EL at 6.2, 7.2, and $9.5 \mathrm{~nm}^{-1}$ and they are $\sqrt{3}$ times, twice, and $\sqrt{7}$ times larger than that of the first peak $(3.6 \mathrm{~nm}$ ${ }^{1}$ ), indicating hexagonal packing of ATBC chains as shown in Figure 8c. Therefore, the distance $a$ between two chains are calculated from the d-spacing to be $2.0 \mathrm{~nm}, 1.6 \mathrm{~nm}$, and $2.1 \mathrm{~nm}$ for ATBC, ATEC, and ATHC, respectively. These values are appreciably larger than $d_{0}$ determined from the phase boundary concentrations in THF, thus reasonable.

The phase boundary concentration $c_{\mathrm{A}}$ between biphasic and liquid crystalline phase may be estimated from the polymer mass concentration of hexagonally packed ATBC chain which is related with $a$ as

$$
c_{\mathrm{A}}=\frac{2 M_{\mathrm{L}}}{\sqrt{3} N_{\mathrm{A}} a^{2}}
$$

Therefore the $c_{\mathrm{A}}$ values are calculated to be $0.86,0.79$, and $0.79 \mathrm{~g} \mathrm{~cm}^{-3}$ for ATBC, ATEC, and ATHC, respectively. These values correspond to the volume fraction $\phi=0.72,0.61$, and 0.70 , which is defined as $\phi=c_{\mathrm{A}} \bar{v}$. They are quite smaller than the ideal volume fraction $\pi / \sqrt{ } 12=$ 0.9069 for hexagonal close-packed cylinder structure. The estimated $c_{\mathrm{A}}$ values are plotted against $M_{\mathrm{w}}$ in Figure 9 along with $c_{\mathrm{I}}$ from Figure 5 and those for THF solution. The biphasic regions of ATBC in ELs are much wider than that for the ATBC-THF system. Another interesting point is that $c_{\mathrm{A}}$ and packing structure in concentrated $\mathrm{D}-\mathrm{EL}$ and $\mathrm{L}_{\mathrm{L}} \mathrm{EL}$ solution are substantially the same whereas $c_{\mathrm{I}}$ in the former solvent is quite smaller than the latter one. This indicates that we may obtain highly concentrated smectic phase from rather dilute ATBC solution of D-EL. To obtain more detailed structure and mechanical properties, liquid crystal films might give us useful information as is the case with a cellulose derivative, ${ }^{40}$ but we did not discuss film properties because reproducible films are hardly obtained due to the droplet formation in concentrated ethyl lactate solutions. 

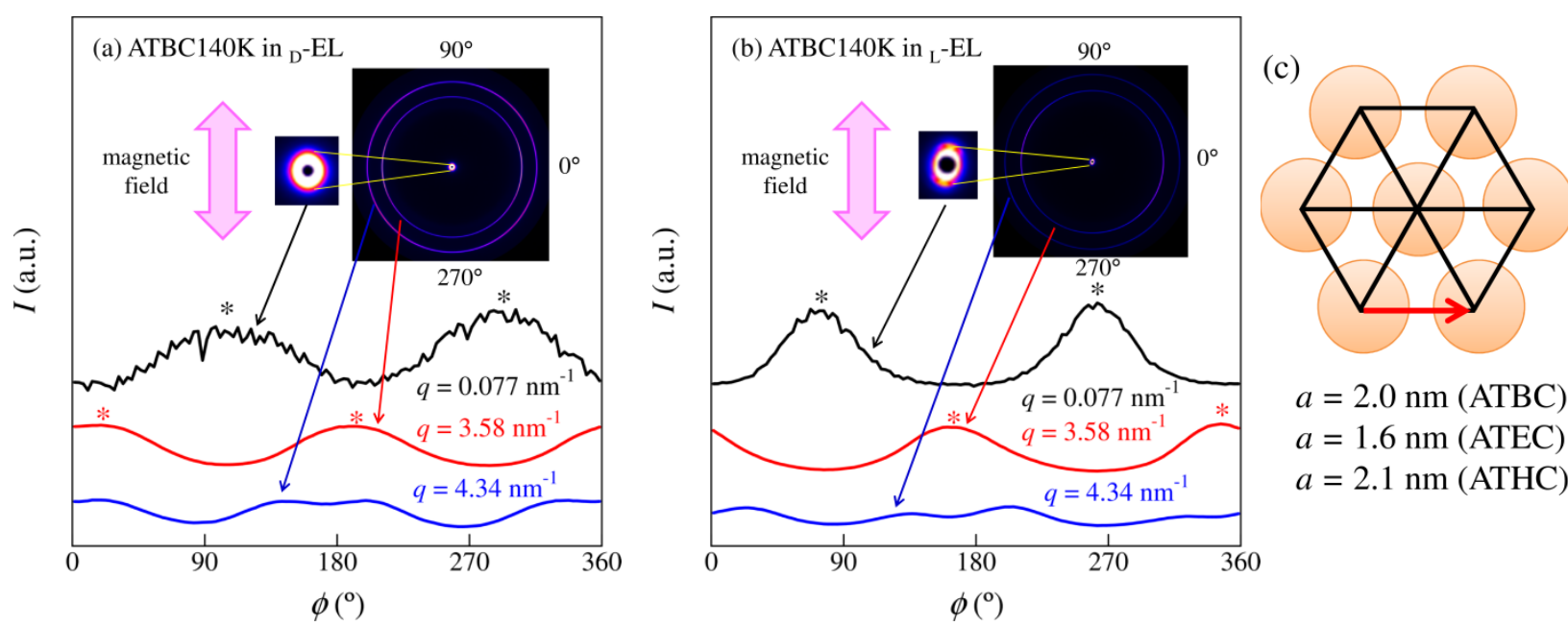

$a=2.0 \mathrm{~nm}(\mathrm{ATBC})$

$a=1.6 \mathrm{~nm}$ (ATEC)

$a=2.1 \mathrm{~nm}(\mathrm{ATHC})$

Figure 8. Azimuth angle dependence of scattering intensities for each diffraction peak for

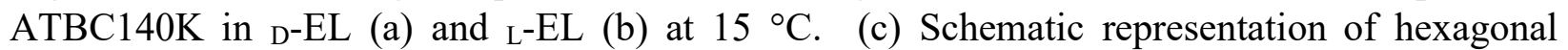
packing of the three amylose alkylcarbamates.

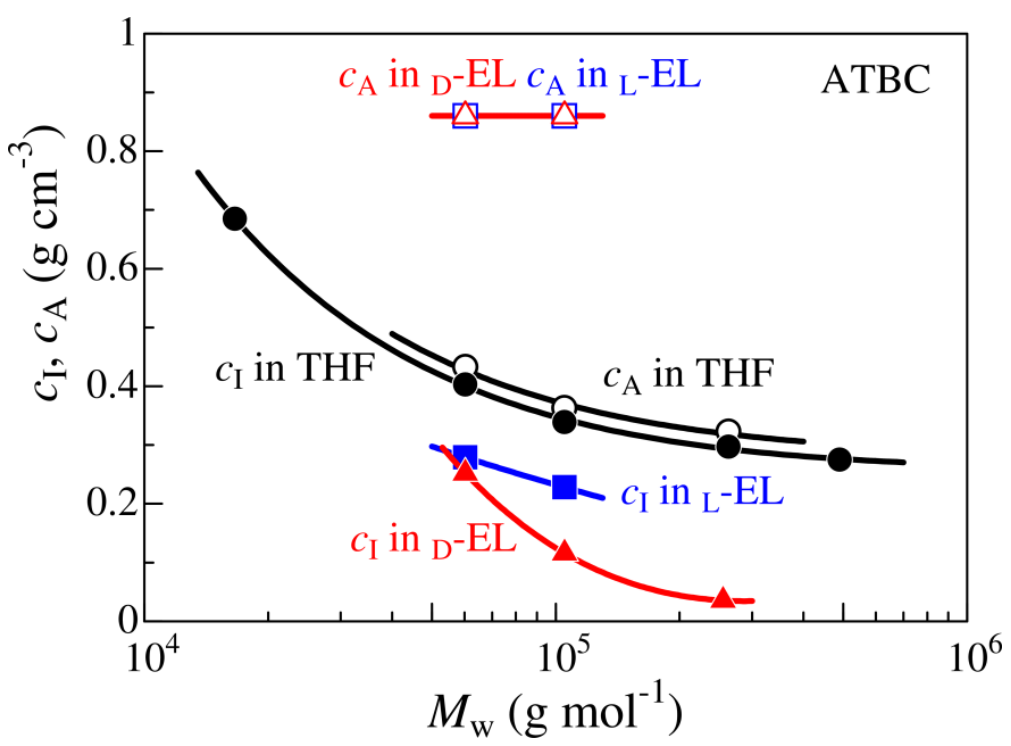

Figure 9. Phase diagram of ATBC in D-EL (triangles), L-EL (squares), and in THF (circles) at 25 ${ }^{\circ} \mathrm{C}$. Unfilled and filled symbols denote $c_{\mathrm{A}}$ and $c_{\mathrm{I}}$, respectively.

Similar diffraction peak of SAXS for ATEC32K in THF, in which the phase boundary concentrations $c_{\mathrm{A}}$ and $c_{\mathrm{I}}$ cannot be explained by the SPT theory as is the case with the amylose alkylcarbamates in ELs, is found at $q=0.17 \mathrm{~nm}^{-1}$ as shown in Figure 10, corresponding to 37 $\mathrm{nm}$ in d-spacing. This value is close to the chain contour length of $31 \mathrm{~nm}$ for ATEC32K. Thus, this suggests smectic order of the sample in THF. It should be noted that such diffraction was not observed for the other amylose derivative samples in THF in the similar concentration range: In other words, only nematic (or cholesteric) phase was found for ATBC, ATEC, and ATHC except for a low molar mass ATEC sample. 


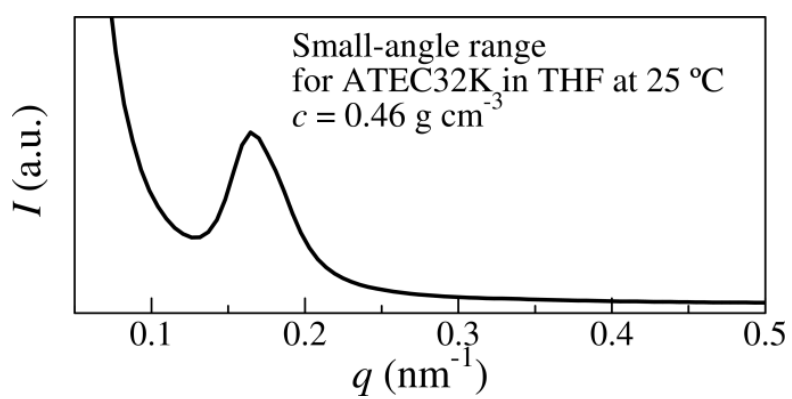

Figure 10. A SAXS profile for ATEC32K in THF at $25^{\circ} \mathrm{C}\left(c=0.46 \mathrm{~g} \mathrm{~cm}^{-3}\right)$.

\section{- CONCLUSION}

Three amylose tris(alkylcarbamate)s, that is, ATBC, ATEC, and ATHC, have lyotropic liquid crystallinity in THF and the phase diagram was successfully explained by the current theory except for the lowest molar mass sample of ATEC. Among them, the cholesteric pitch of ATBC overlaps the wavelength of visible light and it increases with raising temperature and with lowering concentration. On the other hand, the phase diagram of ATBC, ATEC, and ATHC in ethyl lactates is found to be much different from those in THF even though the dilute solution behaviors in these solvents are quite similar. From SAXS measurements, it was found that the anisotropic phase of the three amylose alkylcarbamates in ethyl lactates have smectic order with very high concentration $\left(\sim 0.8 \mathrm{~g} \mathrm{~cm}^{-3}\right)$, which is irrespective of the solvent chirality while the phase boundary concentration between isotropic and biphasic region for ATBC in D-EL solution is appreciably smaller than that in ${ }_{\mathrm{L}}$-EL.

\section{- ASSOCIATED CONTENT}

Supporting Information

A theoretical supplement for the polymer end effect on the cholesteric pitch. This material is available free of charge via the Internet at http://pubs.acs.org.

- Author Information

Corresponding Author

*E-mail:kterao@chem.sci.osaka-u.ac.jp

Notes

The authors declare no competing financial interest.

\section{- Acknowledgments}

The synchrotron radiation experiments were performed at the BL40B2 in SPring-8 with the approval of the Japan Synchrotron Radiation Research Institute (JASRI) (Proposal Nos. 2010A1587, 2011A1049, 2011A1925, and 2011B1068) and at the BL-10C in KEK-PF under the approval of the Photon Factory Program Advisory Committee (No. 2010G080). The authors thank Prof. Tsunehisa Kimura and Dr. Fumiko Kimura (Kyoto Univ.) for magnetic alignment experiments and fruitful discussion, Prof. Kenji Okuyama and Dr. Tatsuya Kawaguchi for preliminary X-ray diffraction measurements, and Prof. Yoji Inoko (Osaka Univ.) for SAXS measurements in KEK-PF. This work was partially supported by JSPS KAKENHI Grant No. 23750128 . 


\section{References}

(1) Hamley, I. W. Soft Matter 2010, 6, 1863-1871.

(2) Werbowyj, R. S.; Gray, D. G. Mol. Cryst. Liq. Cryst. 1976, 34, 97-103.

(3) Werbowyj, R. S.; Gray, D. G. Macromolecules 1980, 13, 69-73.

(4) Patel, D. L.; Gilbert, R. D. J. Polym. Sci., Polym. Phys. Ed. 1981, 19, 1449-1460.

(5) Gilbert, R. D.; Patton, P. A. Prog. Polym. Sci. 1983, 9, 115-131.

(6) Conio, G.; Bianchi, E.; Ciferri, A.; Tealdi, A.; Aden, M. A. Macromolecules 1983, $16,1264-1270$.

(7) Laivins, G. V.; Gray, D. G. Macromolecules 1985, 18, 1753-1759.

(8) Bianchi, E.; Ciferri, A.; Conio, G.; Cosani, A.; Terbojevich, M. Macromolecules 1985, 18, 646-650.

(9) Zugenmaier, P.; Vogt, U. Makromol. Chem. 1983, 184, 1749-1760.

2943.

(10) Sato, T.; Shimizu, T.; Kasabo, F.; Teramoto, A. Macromolecules 2003, 36, 2939-

(11) Wang, L.; Huang, Y. Macromolecules 2003, 37, 303-309.

(12) Nishio, Y. Adv. Polym. Sci. 2006, 205, 97-151.

(13) Rout, D. K.; Barman, S. P.; Pulapura, S. K.; Gross, R. A. Macromolecules 1994, 27, 2945-2950.

(14) Kuse, Y.; Asahina, D.; Nishio, Y. Biomacromolecules 2009, 10, 166-173.

(15) Ochiai, T.; Terao, K.; Nakamura, Y.; Yoshikawa, C.; Sato, T. Polymer 2012, 53, 3946-3950.

(16) Burchard, W. Makromol. Chem. 1965, 88, 11-28.

(17) Burchard, W. In Soft-Matter Characterization; Borsali, R. Pecora, R. ed.; Springer: Berlin, 2008; Vol. 1, p 465-603.

(18) Burchard, W.; Husemann, E. Makromol. Chem. 1961, 44-6, 358-387.

(19) Wenzel, M.; Burchard, W.; Schätzel, K. Polymer 1986, 27, 195-201.

(20) Kasabo, F.; Kanematsu, T.; Nakagawa, T.; Sato, T.; Teramoto, A. Macromolecules 2000, 33, 2748-2756. 201-207.

(21) Terao, K.; Fujii, T.; Tsuda, M.; Kitamura, S.; Norisuye, T. Polym. J. 2009, 41,

(22) Zugenmaier, P.; Voihsel, M. Makromol. Chem., Rapid Commun. 1984, 5, 245-253.

(23) Terao, K.; Murashima, M.; Sano, Y.; Arakawa, S.; Kitamura, S.; Norisuye, T. Macromolecules 2010, 43, 1061-1068.

(24) Terao, K.; Maeda, F.; Oyamada, K.; Ochiai, T.; Kitamura, S.; Sato, T. J. Phys.

Chem. B 2012, 116, 12714-12720.

(25) Sano, Y.; Terao, K.; Arakawa, S.; Ohtoh, M.; Kitamura, S.; Norisuye, T. Polymer 2010, 51, 4243-4248.

(26) Arakawa, S.; Terao, K.; Kitamura, S.; Sato, T. Polym. Chem. 2012, 3, 472-478.

(27) Yu, S. M.; Conticello, V. P.; Zhang, G.; Kayser, C.; Fournier, M. J.; Mason, T. L.; Tirrell, D. A. Nature 1997, 389, 167-170.

(28) Okoshi, K.; Kamee, H.; Suzaki, G.; Tokita, M.; Fujiki, M.; Watanabe, J. Macromolecules 2002, 35, 4556-4559.

(29) Kimura, T. Polym. J. 2003, 35, 823-843.

(30) Sato, T.; Teramoto, A. Adv. Polym. Sci. 1996, 126, 85-161.

(31) Maeno, K.; Nakamura, Y.; Terao, K.; Sato, T.; Norisuye, T. Kobunshi Ronbunshu 1999, 56, 254-259. 
(32) Nakamura, Y.; Koori, M.; Li, Y.; Norisuye, T. Polymer 2008, 49, 4877-4881.

(33) Natsume, T.; Wu, L. B.; Sato, T.; Terao, K.; Teramoto, A.; Fujiki, M.

Macromolecules 2001, 34, 7899-7904.

(34) Sato, T.; Nakamura, J.; Teramoto, A.; Green, M. M. Macromolecules 1998, 31, 1398-1405.

(35) Sato, T.; Teramoto, A. Macromolecules 1996, 29, 4107-4114.

(36) Tanaka, H. Macromolecules 1992, 25, 6377-6380.

(37) Zhang, G. Z.; Wu, C. Adv. Polym. Sci. 2006, 195, 101-176.

(38) Aseyev, V. O.; Tenhu, H.; Winnik, F. M. Adv. Polym. Sci. 2006, 196, 1-85.

(39) Takahashi, R.; Sato, T.; Terao, K.; Qiu, X. P.; Winnik, F. M. Macromolecules 2012, 45, 6111-6119.

(40) Sena, C.; Godinho, M. H.; Oliveira, C. L. P.; Figueiredo Neto, A. M. Cellulose 2011, 18, 1151-1163. 
For Table of Contents Use Only

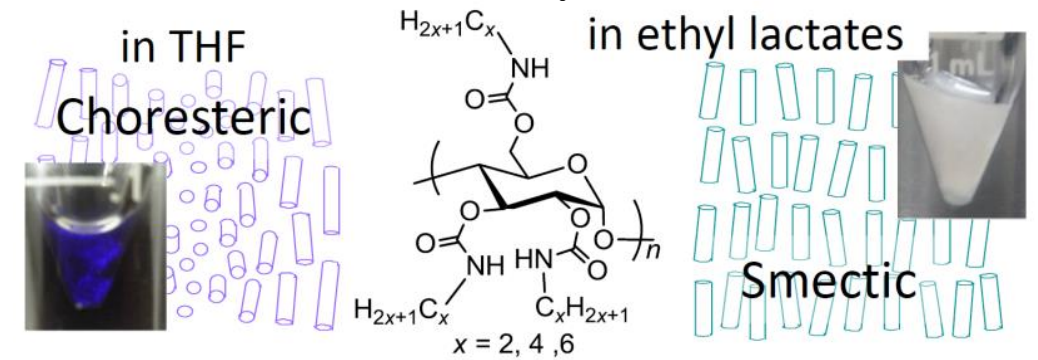

Lyotropic Liquid Crystallinity of Amylose Tris(alkylcarbamates): Cholesteric and Smectic Phase Formation in Different Solvents

Keiko Oyamada, Ken Terao,* Masayori Suwa, Shinichi Kitamura, Takahiro Sato 
Correction to Lyotropic Liquid Crystallinity of Amylose Tris(alkylcarbamates): Cholesteric and Smectic Phase Formation in Different Solvents

Keiko Oyamada, Ken Terao, ${ }^{*}$ Masayori Suwa, Shinichi Kitamura, and Takahiro Sato Macromolecules 2013, 46 (11), 4589-4595. DOI: 10.1021/ma400787c.

The distances $a$ between two chains calculated from the diffraction peaks at $3.65,4.5$, and $3.4 \mathrm{~nm}^{-1}$ for ATBC, ATEC, and ATHC in ethyl lactates should be the same as $d$-spacing, that is, $1.72,1.40$, and $1.85 \mathrm{~nm}$, respectively. Therefore, values of the phase boundary concentration $c_{\mathrm{A}}$ between biphasic region and anisotropic phase in pp. 4593 are 1.15, 1.02, and $1.05 \mathrm{~g} \mathrm{~cm}^{-3}$ and the volume fractions $\phi$ are 0.96, 0.79, and 0.93 for ATBC, ATEC, and ATHC. These values are fairly close or slightly smaller than the ideal volume fraction $\pi / \sqrt{12}=0.9069$ for hexagonal close packed cylinder structure. Regarding with this, collected Figure 9 is illustrated below.

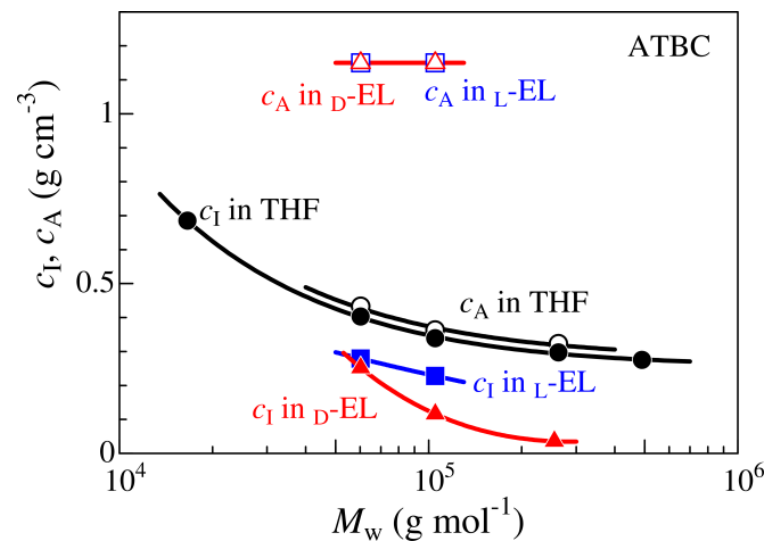

Figure 9. Phase diagram of ATBC in ${ }_{D}$-EL (triangles), ${ }_{\text {-EL }}$ (squares), and in THF (circles) at 25 ${ }^{\circ} \mathrm{C}$. Unfilled and filled symbols denote $c_{\mathrm{A}}$ and $c_{\mathrm{I}}$, respectively. 